\title{
Peripheral immune cell reactivity and neural response to reward in patients with depression and anhedonia
}

Sara Costi ${ }^{1,11}$, Laurel S. Morris ${ }^{1,11}$, Abigail Collins ${ }^{1}$, Nicolas F. Fernandez ${ }^{2}$, Manishkumar Patel (iD ${ }^{2}$, Hui Xie ${ }^{2}$, Seunghee Kim-Schulze ${ }^{2,3}$, Emily R. Stern ${ }^{4,5}$, Katherine A. Collins ${ }^{1,5}$, Flurin Cathomas ${ }^{6}$, Michael K. Parides ${ }^{7}$, Alexis E. Whitton (iD ${ }^{8,9}$, Diego A. Pizzagalli (iD ${ }^{8}$, Scott J. Russo (iD ${ }^{6,10}$ and James W. Murrough (D ${ }^{1,6 凶}$

This is a U.S. government work and not under copyright protection in the U.S.; foreign copyright protection may apply 2021

Increased levels of peripheral cytokines have been previously associated with depression in preclinical and clinical research. Although the precise nature of peripheral immune dysfunction in depression remains unclear, evidence from animal studies points towards a dysregulated response of peripheral leukocytes as a risk factor for stress susceptibility. This study examined dynamic release of inflammatory blood factors from peripheral blood mononuclear cells (PBMC) in depressed patients and associations with neural and behavioral measures of reward processing. Thirty unmedicated patients meeting criteria for unipolar depressive disorder and 21 healthy control volunteers were enrolled. PBMCs were isolated from whole blood and stimulated ex vivo with lipopolysaccharide (LPS). Olink multiplex assay was used to analyze a large panel of inflammatory proteins. Participants completed functional magnetic resonance imaging with an incentive flanker task to probe neural responses to reward anticipation, as well as clinical measures of anhedonia and pleasure including the Temporal Experience of Pleasure Scale (TEPS) and the Snaith-Hamilton Pleasure Scale (SHAPS). LPS stimulation revealed larger increases in immune factors in depressed compared to healthy subjects using an aggregate immune score $\left(t_{49}=2.83, p=0.007\right)$. Higher peripheral immune score was associated with reduced neural responses to reward anticipation within the ventral striatum (VS) $(r=-0.39, p=0.01)$, and with reduced anticipation of pleasure as measured with the TEPS anticipatory sub-score $(r=-0.318, p=0.023)$. Our study provides new evidence suggesting that dynamic hyper-reactivity of peripheral leukocytes in depressed patients is associated with blunted activation of the brain reward system and lower subjective anticipation of pleasure.

Translational Psychiatry (2021)11:565; https://doi.org/10.1038/s41398-021-01668-1

\section{INTRODUCTION}

Major depressive disorder (MDD) is a common, chronic and debilitating condition characterized by affective, cognitive, and behavioral symptoms [1, 2]. Despite its large health impact, the neurobiological mechanisms underlying this disorder are still largely unknown. Anhedonia, the reduced ability to experience pleasure, is a core symptom of this disorder and is associated with reduced neural response to reward, poor response to antidepressant medication, and increased risk for suicide [3-5].

Convergent lines of research suggest a dysfunctional immune response in the context of clinical depression [6-9], more specifically linked to anhedonia [10,11]. Over one-third of patients treated with interferon alpha develop significant depressive symptoms and anhedonia [12, 13] and several studies and meta-analyses have reported an association between depression and increased circulating levels of pro-inflammatory cytokines, most consistently interleukin-6 (IL-6) and tumor necrosis factor alpha (TNF-alpha) [14-16]. Further, there is evidence that childhood adversity, a well-established risk factor for depression [17], is associated with long-term inflammation [18, 19].

Recent work has also connected peripheral immune dysfunction to brain systems that mediate key aspects of depression, such as reward processing $[20,21]$. Despite this evidence, the current data appears heterogenous and the precise nature of inflammatory dysregulation in the context of depression remains unclear. Preclinical data show that levels of peripheral cytokines predict the development of anhedonic-like behavior [10]. Repeated exposures to social defeat stress (a well-validated animal model of depression) in rodents causes anhedonia-like behavior characterized by social avoidance and reduced interest in reward in a subset of mice ('susceptible' mice); in contrast, 'resilient' mice do not develop such behavior. In this model, cytokine profiles 20 min following the first exposure to the stressor (an aggressor mouse) showed that IL- 6 was significantly elevated in animals that later

\footnotetext{
${ }^{1}$ Depression and Anxiety Center for Discovery and Treatment, Department of Psychiatry, Icahn School of Medicine at Mount Sinai, New York, NY, USA. ${ }^{2}$ Human Immune

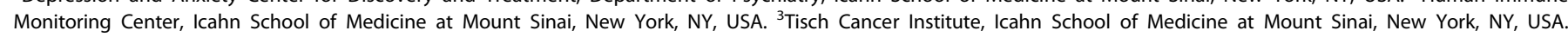

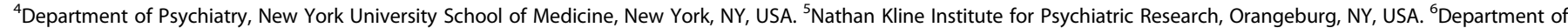

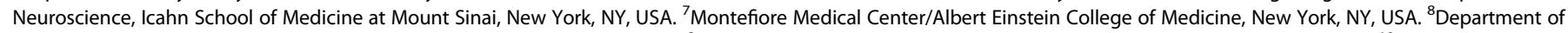

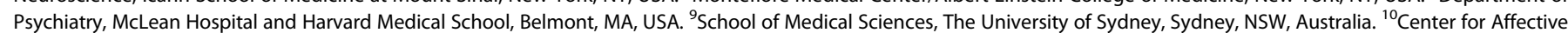

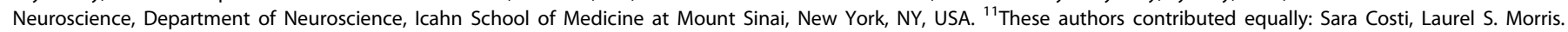
email: james.murrough@mssm.edu
} 
exhibited the susceptible phenotype compared with control and resilient mice. Leukocytes collected prior to stress exposure from animals that later developed a susceptible phenotype revealed a significant increase in IL-6 in response to ex vivo stimulation with the bacterial endotoxin lipopolysaccharide (LPS) compared to animals that showed a resilient phenotype [10]. LPS is a major constituent of the membrane of gram-negative bacteria and strongly stimulates innate immunity in diverse species, including humans [22]. LPS, acting as an agonist on Toll-like receptors (TLR)4 localized on the surface of myeloid cells, induces a dynamic response leading to the production of inflammatory cytokines and chemokines. While previous studies have shown that LPS administration is capable of inducing depressive-like symptoms [23] in healthy subjects, the specific question of leukocyte cell reactivity to an immune challenge in depressed subjects has been understudied. Interestingly, in vitro lymphocytic activity has been previously explored in the context of depression with depressed subjects showing a decrease in vitro lymphocyte activity [24].

Building on these data, the current study aimed to translate preclinical findings into humans by investigating a large panel of peripheral immune factors in subjects with depression from peripheral blood mononuclear cells (PBMC) stimulated ex vivo with LPS. To test specific immune-behavior relationships with anhedonia, subjects with unipolar depression and healthy volunteers completed a multilevel assessment of anhedonia using rating scales, a computer-based task [probabilistic reward task (PRT) $[25,26]]$, and a task-based functional magnetic resonance imaging (fMRI) measure of reward anticipation (incentive flanker task, IFT) [27-29], with a particular focus on the ventral striatum (VS). We examined if LPS stimulation revealed larger increases in immune factors in depressed subjects compared to healthy volunteers. We further explored whether LPS-stimulated immune factors were associated with objective and self-reported measures of anhedonia and neural reactivity to reward anticipation.

\section{MATERIALS AND METHODS \\ Study participants and design}

The study was conducted at the Icahn School of Medicine at Mount Sinai (ISMMS) in New York City wherein participants were recruited between September 2016 and August 2018. The institutional review board at ISMMS approved the study, and written informed consent was obtained prior to any study procedure. Participants were between the ages of 18 and 55 and met DSM-5 criteria for a primary diagnosis of major depressive disorder (MDD), persistent depressive disorder (PDD), or other specified depressive disorder, as assessed by the Structured Clinical Interview for DSM-5 Axis I Disorders - Patient Edition (SCID-I/P) [30]. A healthy control (HC) group with similar age, sex, and body mass index (BMI) was also enrolled, as these variables are well-known to affect inflammatory markers [31-37]. Exclusionary criteria included a history of inflammatory or autoimmune disorder, clinically significant abnormalities of laboratories, and any unstable medical condition. Treatment with psychotropic medication or systemic steroids within 4 weeks of assessment, or use of medication or nutritional supplement known to affect the immune system within one week of the assessment visit, were exclusionary. A complete list of the inclusion and exclusion criteria is available in Supplemental Material.

During the screening visit, medical and psychiatric history was obtained. All participants underwent clinical laboratory tests and toxicology screening. A pregnancy test was performed in premenopausal women. Within 4 weeks of screening, all eligible participants completed the assessment visit, which included a blood draw for inflammatory markers collection, and clinical and neuro-behavioral assessment of depression and anhedonia.

\section{Clinical assessment of anhedonia}

Anhedonia was assessed using the Snaith-Hamilton Pleasure Scale (SHAPS) [38], a well-validated self-report questionnaire assessing pleasure capacity, both consummatory and anticipatory. Hedonic capacity was also measured using the Temporal Experience of Pleasure Scale (TEPS) [39], a validated self-report that provides specific sub-scores of anticipatory and consummatory pleasure. A higher score of the SHAPS indicates more severe anhedonia; a higher score on the TEPS indicates greater pleasure and hence lower anhedonia severity.

\section{Blood collection and processing}

Blood was collected between 8 AM and 10 AM into tubes containing ACD Solution $\mathrm{A}$ as anti-coagulant (BD Vacutainer) and delivered to the Human Immune Monitoring Center (HIMC) of ISMMS for processing and storage. To mitigate the effect of confounding factors, participants were asked to reduce the intake of lipids and to avoid alcoholic beverages in the 12 and $24 \mathrm{~h}$, respectively, prior to the blood draw. Details on the blood processing procedures are available in Supplemental Material.

The samples were analyzed mid-way through the study and at the end of the study period using Olink multiplex assay - Inflammatory panel (Olink Bioscience, Uppsala, Sweden), according to the manufacturer's instructions. The inflammatory panel allows the detection of 92 proteins associated with human inflammatory conditions. More details are included in the Supplemental Material.

\section{MRI acquisition, processing, and reward task (incentive flanker task)}

All MRI data were acquired with a Siemens 3T MAGNETOM Skyra scanner and a 32-channel head coil at ISMMS. Functional scans were preprocessed and denoised for motion and physiological noise using multi-echo independent component analysis (ME-ICA) [40, 41]. Multi-echo functional MRI data were decomposed into independent components, and scaled against TE [40-42]. Components with high TE-dependence are considered BOLD-like whereas components with low TE-dependence are considered noise-like [40-42]. Removal of non-BOLD components allows robust data denoising for motion, physiological, and scanner artifacts [42]. The incentive flanker task (IFT) is a modification of the monetary incentive delay (MID) task [29]. Further details, including a detailed description of the task, are included in the Supplemental Material (Fig. S1).

\section{Probabilistic reward task}

The probabilistic reward task (PRT) [25] is a signal detection test that provides an objective assessment of reward learning and was completed on the assessment visit. A detailed description of the task is provided in the Supplemental Material.

\section{Statistical analyses}

The sample size was calculated using anhedonia as a continuous outcome and relying on preliminary data wherein the magnitude of association between a linear combination of immune factors provided $>90 \%$ power to detect an association between immune factors and anhedonia based on a proposed sample size of $n=50$ (MDD, $n=30 ; \mathrm{HC}, n=20$ ).

Demographic and clinical characteristics, blood biomarkers, and behavioral measures of reward are summarized separately. Continuous variables are summarized using means and standard deviations, while categorical variables are summarized as proportions. Cytokines and chemokines with $>50 \%$ missing or below the limit of detection (LOD) values were excluded from the analysis. Outliers, defined as standard deviations $>3$, were excluded separately for each immune factor [43]. Each of the remaining proteins had $<5 \%$ missing data, considered randomly missing. Predictive mean matching was used for imputation of missing data using multivariate imputation by chained equations (MICE) using R software [44], which computes a realistic distribution, separately optimized for each variable, and randomly selects a regression-predicted value from 50 iterations [45-47]. A list of analytes that were not included in the analysis is available in the Supplemental Material. To determine the influence of stimulated levels of immune factors, the proteins' fold change was calculated as the value of the cytokine released by PBMC following LPS stimulation divided by the un-stimulated value of the cytokines secreted by PBMC prior to the LPS challenge. The resultant fold-change protein data (42 proteins $\times 51$ subjects) were entered into principal component analysis (PCA) to reduce the dimensionality of the multidimensional data, while retaining the majority of variability within the data. A scree plot of eigenvalues was computed and inspected to indicate component significance (see Supplemental Material). Significant components from the PCA were examined for group differences based on individual factor loadings using independent-samples $t$-test, and used for correlations with self-report questionnaires, clinician-administrated scales, measures of reward learning, and brain activation using two-tailed 
Pearson's correlation. The PCA was used for the statistical analysis and results.

Task-based fMRI analysis included first-level general linear models (GLM) with regressors for cue onset (reward/loss/neutral), flanker onset, and feedback onset, each including duration modulation using AFNI's stim_DM function, and convolved with the hemodynamic response function. The contrast of interest for reward anticipation was gain cue $>$ neutral cue. Activation for each contrast was extracted within the VS ROI from FSL's Harvard-Oxford atlas [48] for each subject and entered into independent samples $t$-test for group difference comparison. Pearson correlation was used to examine relationships between VS activation during reward anticipation and significant immune principal components. An additional whole-brain analysis was performed using two-tailed Pearson's correlation between whole-brain activation maps for gain $>$ neutral cue and the first significant immune principal component (PC1) factor loading. For this, a voxelwise, cluster-defining threshold of $p<0.005$ was used plus clusterwise correction of $\mathrm{K}>60$ as calculated by AFNI's ACF function to mitigate against spatial autocorrelation and the incidence of false-positives [49].

\section{RESULTS}

\section{Sample characteristics}

Of the 57 subjects assessed for eligibility, 51 met inclusion/ exclusion criteria and completed the assessment visit: 30 subjects with depression and 21 matching healthy volunteers. Within the depressed group, 28 subjects met criteria for current MDD, one for PDD, and one for other specified depressive disorder. Of these, 28 were in a current major depressive episode (MDE). No significant difference emerged between the two groups in sociodemographic characteristics, age, gender, BMl, or smoking status (Table 1).

\section{Immune markers}

Basal plasma factors: Analysis of peripheral immune parameters were conducted utilizing 69 analytes from plasma. There were no group differences in circulating plasma levels of inflammatory factors (see Supplemental Material; Table S1).

LPS simulated PBMCs: Analysis of peripheral immune parameters was conducted utilizing 42 analytes from supernatant from LPS-stimulated PBMCs, based on the LOD described above. In contrast to the findings with basal plasma levels, patients with depression showed a greater increase in PBMC release of inflammatory factors following stimulation with LPS compared to healthy controls as calculated by the immune markers' fold change. Immune factors fold change in depressed and healthy controls are reported in Fig. $1 \mathrm{~A}$.

LPS-stimulated PBMC analytes were subjected to principal component analysis (PCA). The first three components preceded the scree plot eigenvector elbow, all had an eigenvector value $>1$ and were thus considered significant. Principle component 1 (PC1) explained a large proportion of the variance $(52.8 \%)$, with an eigenvector of 12.6 (Fig. S2). PC1 included highest factor loadings for TNF, C-C motif chemokine 20 (CCL20), IL6, interleukin-12 receptor subunit beta (IL12B), and monocyte-chemotactic protein 3 (MCP3) (Fig. 1B). This component was significantly higher in patients with depression compared to controls $\left(t_{49}=2.83, p=\right.$ 0.007 , Fig. 1C). The other two significant components, PC2 and PC3 had highest loadings for TNF and IL-12b, respectively, and were not different between groups ( $p^{\prime} s>0.3$ ).

\section{Correlation between immune factors and clinical symptoms}

The relationship between peripheral immune factors and anhedonia was examined across the full sample, and within the two groups separately. For these correlations, BMI, age, and sex were included as covariates. A greater PC1 score was associated with greater anticipatory anhedonia as measured by the TEPS anticipatory sub-score $(r=-0.34, p=0.016)$ (Fig. 2). PC1 was not correlated with the TEPS consummatory anhedonia as measured by the TEPS consummatory sub-score $(r=-0.12, p=0.170)$ or
Table 1. Demographic and clinic characteristic of study sample.

\begin{tabular}{|c|c|c|c|}
\hline & $\operatorname{MDD}(N=30)$ & $\begin{array}{l}\text { Healthy } \\
\text { controls } \\
(N=21)\end{array}$ & $p$ value \\
\hline Age, $M(\mathrm{SD})$ & $37.1(10.8)$ & $37.1(9.6)$ & 0.98 \\
\hline Gender (male), $n$ (\%) & $14(46.7)$ & $13(61.9)$ & 0.28 \\
\hline $\mathrm{BMI}, M(\mathrm{SD})$ & $26.7(5.8)$ & $24.8(4.7)$ & 0.22 \\
\hline Daily smoking, $n(\%)$ & $0(0)$ & $0(0)$ & 1 \\
\hline hs-CRP level, $M$ (SD) & $3.8(7.2)$ & $1.7(1.6)$ & 0.19 \\
\hline \multicolumn{4}{|l|}{ Race, $n$ (\%) } \\
\hline White/Caucasian & $16(53.3)$ & $10(47.6)$ & 0.08 \\
\hline \multicolumn{4}{|l|}{ Ethnicity, $n$ (\%) } \\
\hline Hispanic/Latino & $7(23.3)$ & $3(14.3)$ & 0.22 \\
\hline \multicolumn{4}{|l|}{ Employment, $n$ (\%) } \\
\hline Employed & $21(70.0)$ & $17(81.0)$ & 0.05 \\
\hline \multicolumn{4}{|l|}{ Education, $n$ (\%) } \\
\hline Bachelor Degree & $21(70.0)$ & $16(76.2)$ & 0.61 \\
\hline \multicolumn{4}{|l|}{ Relationship status, $n$ (\%) } \\
\hline Married & $2(6.7)$ & $4(19.1)$ & 0.07 \\
\hline \multicolumn{4}{|l|}{ Psychiatric comorbidities } \\
\hline $\begin{array}{l}\text { Anxiety disorder, } \\
n(\%)\end{array}$ & $14(46.7)$ & $0(0)$ & $<0.001$ \\
\hline PTSD current, $n$ (\%) & $1(3.3)$ & $0(0)$ & 0.22 \\
\hline MADRS, $M(\mathrm{SD})$ & $27.4(6.4)$ & $1(1.6)$ & $<0.001$ \\
\hline SHAPS, $M$ (SD) & $34.5(7.6)$ & $17.6(4.9)$ & $<0.001$ \\
\hline $\begin{array}{l}\text { TEPS anticipatory, } \\
M \text { (SD) }\end{array}$ & $26.7(10.5)$ & $45.1(6.5)$ & $<0.001$ \\
\hline $\begin{array}{l}\text { TEPS consummatory, } \\
M(\mathrm{SD})\end{array}$ & $26.1(10.5)$ & $39.3(6.1)$ & $<0.001$ \\
\hline
\end{tabular}

Race and ethnicity were reported by the study participants.

$B M l$ body mass index, $h s-C R P$ high-sensitivity $C$ reactive protein, $M$ means, MADRS Montgomery-Åsberg Depression Rating Scale, MDD Major Depressive Disorder, PTSD posttraumatic stress disorder, SD standard deviation, SHAPS Snaith-Hamilton Pleasure Scale, TEPS Temporal Experience of Pleasure Scale.

with general anhedonia as measured by the SHAPS $(r=0.25$, $p=0.071$ ).

\section{Task-based MRI results}

Functional MRI data during the IFT were available for 28 depressed subjects (age $=36.5 \pm 11,14$ females) and $20 \mathrm{HC}$ subjects (age $=$ $37.8 \pm 9.4,7$ females). One MDD subject was excluded due to excessive motion during MRI. Groups did not differ in their performance accuracy $(\mathrm{MDD}=85.9 \% \pm 10.4, \mathrm{HC}=87.7 \% \pm 0.1$, $p=0.76$ ), or their baseline reaction times (MDD $=927.6 \mathrm{~ms} \pm$ 172.5, $\mathrm{HC}=859.3 \mathrm{~ms} \pm 145.7, \quad p=0.16)$. Patients with MDD showed lower VS response to reward (gain cue $>$ neutral cue) compared to controls across all four runs of the task $\left(t_{45}=-1.88\right.$, $p=0.066)$ but most notably during the first run $\left(t_{45}=-2.05, p=\right.$ 0.047), consistent with previous reports of VS habituation to reward over time [50] (Fig. 3). Therefore, follow-up analyses were performed utilizing the first run only.

Consistent with our hypothesis, a higher PC1 immune score was associated with reduced activation to reward anticipation in the VS across both groups $(r=-0.376, p=0.011$; Fig. 3$)$. This association remained significant when controlling for age, gender, and BMI $(r=-0.389, p=0.011)$. No correlation emerged within the MDD $(r=-0.26, p=0.230)$ or HC group $(r=-0.3, p=0.250)$ alone. An additional exploratory whole-brain analysis correlating 
A.

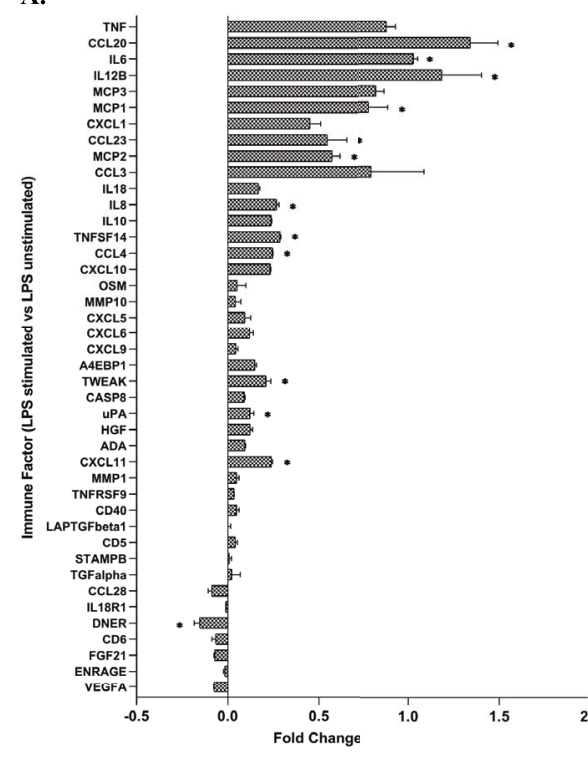

B.

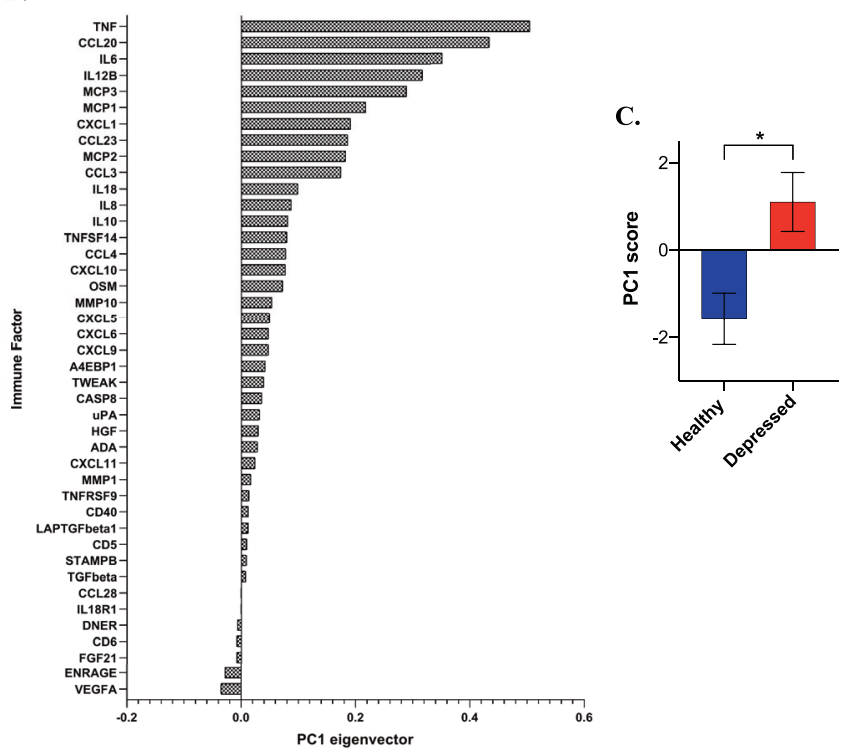

Fig. 1 Heightened peripheral blood mononuclear cells reactivity in depression. A Difference in fold change of levels of lipopolysaccharide (LPS) stimulated immune factors released ex vivo by peripheral blood mononuclear cells (PBMCs) between depressed and healthy volunteers uncorrected $t$-test. B Principal component 1 (PC1) factor loading. PC1 explained a large proportion of the variance, with an eigenvector of 12.6, accounting for $52.8 \%$ of the total variance in the data and included highest factor loadings for tumor necrosis factor alpha (TNF-alpha), chemokine ligand 20 (CCL-20), interleukin-6 (IL-6), interleukin-12 subunit beta (IL-12B), and monocyte-chemotactic protein 3 (MCP3). C Immune reactivity PC1 score difference between depressed subjects and healthy volunteers; ${ }^{*} p<0.05$, error bars represent standard error of the mean (SEM).

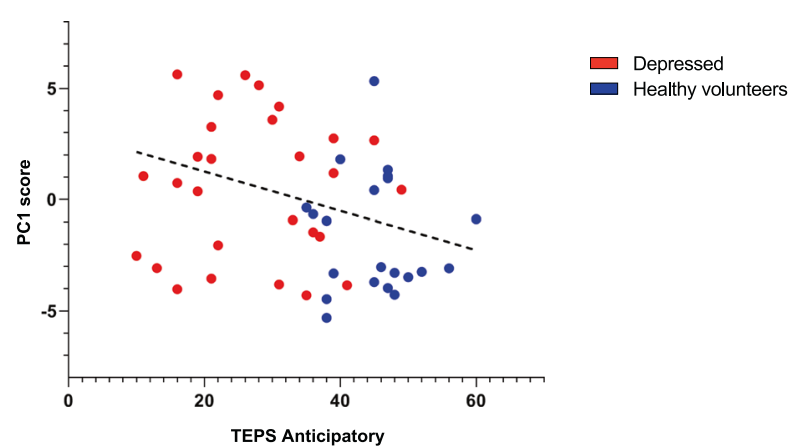

Fig. 2 Peripheral blood mononuclear cells reactivity is negatively associated with response to pleasure. Negative correlation between immune factors released by peripheral blood mononuclear cells (PBMCs) stimulated ex vivo with lipopolysaccharide (LPS) represented by factor loading on PC1 and anticipatory reward, as measured with the anticipation subscale of the TEPS self-report.

PC1 against whole-brain activation to gain > neutral cue revealed no significant correlation between brain activation and PC1.

\section{Behavioral measures of reward (PRT)}

Fifty-one subjects completed the PRT on the assessment day. Of these, 17 healthy volunteers and 23 depressed subjects had valid data following the quality check $(\mathrm{QC})$. Reasons for exclusion were: too many outliers $(n=8)$, uncompleted task $(n=1)$, and below chance accuracy $(n=2)$. The remaining analysis was conducted only on the subjects who passed the QC $(n=40)$.

As hypothesized and consistent with prior reports, response bias (computed by subtracting the total response bias in the first block from the total response bias in the last block) was higher in healthy compared to depressed $\left[t_{38}=2.27 p=0.029\right.$ ); Fig. S3]. Reward learning was associated with greater activation to reward anticipation in the VS $(r=0.32, p=0.053)$, but did not correlate with PC1 immune score upon controlling for BMI, age, and sex $(r=-0.16, p=0.33)$. For more details, see Supplemental Material.

\section{DISCUSSION}

The current study investigated the relationship between inflammatory factors released by PBMCs stimulated ex vivo with LPS, anhedonia and neural reactivity to reward anticipation in depression. We found that depressed subjects showed an exaggerated response to the immune challenge with LPS compared to HC. Further, an increase in immune factors released by PBMCs following LPS stimulation was associated with reduced anticipation of pleasure as measured with the anticipatory subscore of the TEPS and with a reduced response to reward anticipation within the VS in the brain.

Depressed subjects showed increased levels of immune markers released by PBMCs stimulated ex vivo with LPS, consistent with previous work showing similar results in rodent stress models of depression [10]. Here, we were able to translate these findings into humans and expand it through the analysis of a broad range of immune factors. Of note, other authors have investigated the effect of LPS stimulation within depressed subjects with mixed results. A large study [51] using data from the Netherlands Study of Depression and Anxiety (NESDA) on adults with current or remitted depression or anxiety disorders and healthy controls explored the correlations between clinical symptoms of anxiety and depression and inflammatory markers that were assayed from plasma and after whole blood stimulation with LPS. In this large cohort, LPS-stimulated inflammatory markers, but not plasma factors, were associated with anxiety symptom, but not with depression severity, upon adjustment for health condition and lifestyle. A recent study [52] on adolescents with anhedonia across a broad spectrum of psychiatric disorders showed no group differences in cytokine levels following whole blood LPS stimulation. Of note, the different LPS stimulation approach (whole blood compared to isolated PBMC stimulation) 

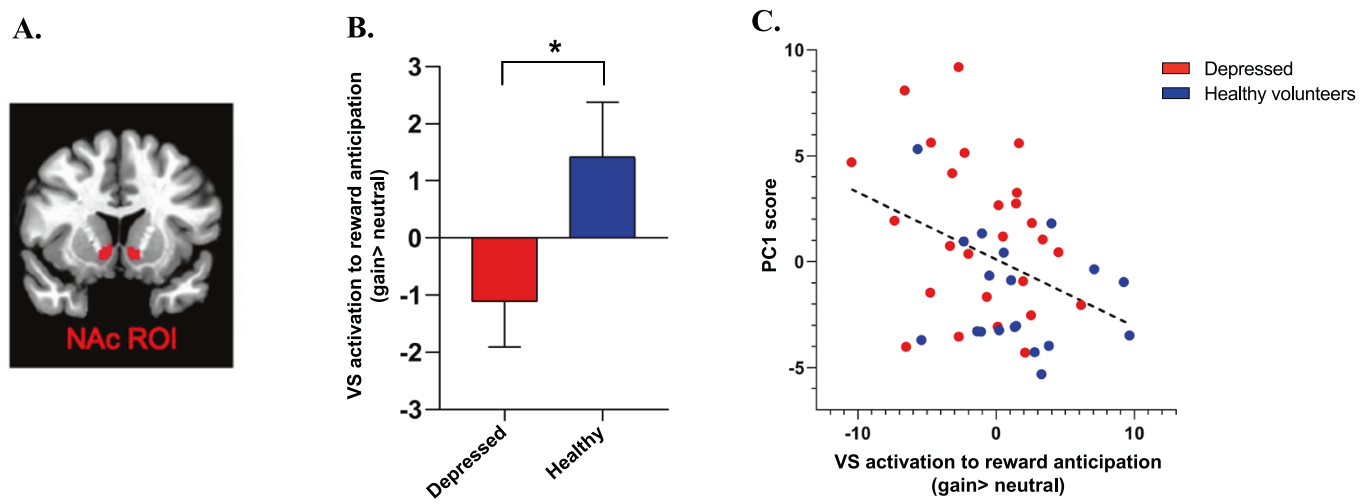

Fig. 3 Blunted response to reward in depression and association with immune reactivity. A Ventral striatum (VS) region of interest (ROI); B Group difference in VS response to reward (gain > neutral cue) during the first run of the task in MDD and HC in VS ROI. C Correlation between immune reactivity, as computed through the principal component 1 (PC1) factor loading, and VS activation to reward (gain cue > neutral cue) on the first run of the (incentive flanker task) IFT across the sample.

may explain the different results. Further, we enrolled only adult subjects diagnosed with unipolar depressive disorders to minimize heterogeneity $[11,53]$. Others [54] analyzed the levels of pro-inflammatory cytokines IL-6, TNF-alpha, and IL-1beta following LPS stimulation on isolated PBMC on depressed and healthy volunteers reporting a reduced response to LPS stimulation in depressed. The authors isolated CD14 + monocytes prior to culture cultured them with LPS and used a chemiluminescence method to measure the levels of the proinflammatory cytokines IL- 6 , TNF-alpha, and IL-1beta. Different LPS stimulation protocol and analysis methods may account for the dissimilar findings.

We did not find a difference between groups in un-stimulated plasma level in the current study. This negative result is discordant with several studies and meta-analyses showing higher levels of some immune factor in patients with depression compared to non-depressed controls, such as C-reactive protein (CRP), IL-6, and TNF-alpha [14, 15, 55]. Notwithstanding a number positive reports, there have been several negative reports as well, including the large NESDA study discussed above. Indeed meta-analyses suggest that findings of elevated circulation inflammatory factors in patients with depression may be small and quite variable $[55,56]$. Recent data suggest that inflammation may be prominent only in a subset of depressed patients and mostly among subjects with treatment resistant depression [57]. A recent study by Syed et al. suggests that lifetime treatment with antidepressant may affect cytokines concentration in MDD [58]. In this work, drugnaive depressed subjects showed an elevation in plasma concentration of pro- and anti-inflammatory cytokines compared to healthy volunteers that was immunosuppressive on freshly isolated PBMCs from a healthy volunteer donor at the monocytes/ dendritic cells, B cells and T cell memory level. Further, a 12-week period treatment with escitalopram, duloxetine or cognitive behavioral therapy (CBT) was associated with an increase in antiinflammatory cytokine levels in both responder and nonresponders, whereas pro-inflammatory cytokines continued to increase in non-responders while they were stabilized in responders.

This study is among the firsts to use Olink inflammatory panel to explore a wide range of inflammatory markers within a psychiatric population [59]. The use of this assay, one of the most extensive panel available for proteins associated with inflammation and related biological processes, highlighted the potential contribution of a broad range of immune and inflammatory factors to depression and anhedonia, beyond well-studied proinflammatory cytokines such as TNF-alpha and IL-6. Interestingly, there has been increasing interest regarding the role of chemokines in the neurobiology of depression [60]. Chemokines act as chemiotactic factors and activators of peripheral immune cells that can potentially contribute to the neurodegeneration observed in depression [61, 62]. For instance, monocytechemotactic proteins (MCP)-1, together with MCP-2 and MCP-3, is well known to exert potent pro-inflammatory action and has been shown to possess the ability to translocate across the blood-brain barrier (BBB) and induce chemotaxis of circulating leukocytes [63]. Thus, broader spectrum analysis of immune markers should be explored to investigate further the biological mechanisms of immune dysregulation in depression.

The second main finding of our study was that the degree of immune cell reactivity was positively associated with impairment in the ability to experience pleasure across groups as measured by self-report. Consistent with the work from Freed et al. [52], the magnitude of immune response was negatively associated with anhedonia severity. Similarly, others investigated the relationship between immune factors released by whole blood LPS stimulated and symptom severity [64] across a spectrum of depressive disorders and showed an association between LPS-stimulated inflammation and depression severity.

Our third finding was that the magnitude of immune cell reactivity was negatively associated with VS activation to reward anticipation. There is substantial evidence that peripheral cytokines impact reward processing areas implicated in anhedonia and depression [65-68]. The administration of pro-inflammatory cytokines (e.g., INF-a) or endotoxin have been shown to reduce neural responses to reward $[67,68]$, while the relationship between plasma high-sensitivity CRP (hs-CRP) and the brain reward circuit has been investigated in depression, at the level of resting-state functional connectivity $[21,65]$. Herein, we showed that greater immune activity was associated with reduced activation to reward anticipation in the VS. These findings suggest immune reactivity in depression may be associated with a specific aspect of the hedonic experience that relies on the brain reward system centered on the VS $[39,69]$. However, caution should be taken in generalizing these results given the limited sample size of this study and potential limitations of test-retest reliability of taskbased fMRI [70]. Further work focusing on the interaction between the peripheral immune system and the brain reward circuit in larger samples are warranted to improve our understanding on the effect of immune dysregulation on the brain circuit of depressed subjects with anhedonia.

Finally, in the current study, depressed subjects were characterized by a blunted reward learning toward rewards as measured by the PRT, although this was not associated with LPS-stimulated immune factors. This is the first report 
investigating the relationship between reward learning (as measured with a computer-based task) and cytokine change following PBMCs stimulation with LPS. A recent study exploring the effect of the influenza vaccination on reward learning in 41 young healthy subjects showed that increases in IL-6 in circulation predicted increased performance on the PRT [71]. The different study population and the mild systemic inflammatory response elicited by the influenza vaccine may explain the differences in behavioral sensitivity to inflammation in this sample compared to healthy volunteers.

Limitations of the study include the relatively small sample size and the enrollment of subjects with a broader unipolar depressive phenotype. The small sample size may have led to Type II errors that could explain, for example, the lack of association between LPS-stimulated immune factor and activation to reward anticipation in the depressed group alone. Hence, this data may guide sample size calculation for future larger studies studying dynamic changes in the immune factors and response to reward within depression. Moreover, this study did not explore the effect of antidepressant medication on immune challenged PBMCs. Although limiting the external validity of the study, given the size of the sample analyzed, we opted to limit enrollment to subjects free of medication due to the unknown interactions between CNS medications on immune factors released by PBMC following LPS stimulation and the well-established effects on antidepressants on fMRI measures in depression. Further, since this study focused on currently depressed subjects and lacks a depressed control group in remission, it is unclear if the altered immune profile observed should be considered a state or a trait characteristic of depression. Finally, the current study focused on the isolation of PBMC prior to LPS stimulation, but did not explore the contribution of cellular immune markers. The increased levels of immune markers released by PBMCs stimulated ex vivo with LPS in depressed compared to healthy controls warrants further exploration and suggest a prominent role for the monocyte/ macrophage lineage in the context of depression. Future studies investigating the cellular mechanisms associated with depression using a transcriptome approach to examine the molecular mechanisms of peripheral inflammation relevant to depression are needed.

In the current study, we found that changes in immune factors in response to LPS stimulation were associated with depression and correlated with impaired anticipatory reward function at the clinical and neuronal level. Future studies with larger sample sizes are required to improve the knowledge of the neurobiological mechanism underlying depression.

\section{REFERENCES}

1. Kessler RC, Petukhova M, Sampson NA, Zaslavsky AM, Wittchen H-U. Twelvemonth and lifetime prevalence and lifetime morbid risk of anxiety and mood disorders in the United States. Int J Methods Psychiatr Res. 2012;21:169-84.

2. Greenberg PE, Fournier A-A, Sisitsky T, Pike CT, Kessler RC. The economic burden of adults with major depressive disorder in the United States (2005 and 2010). J Clin Psychiatry. 2015;76:155-62.

3. Wardenaar KJ, Giltay EJ, van Veen T, Zitman FG, Penninx BWJH. Symptom dimensions as predictors of the two-year course of depressive and anxiety disorders. J Affect Disord. 2012;136:1198-203.

4. Spijker J, de Graaf R, Ten Have M, Nolen WA, Speckens A. Predictors of suicidality in depressive spectrum disorders in the general population: results of the Netherlands Mental Health Survey and Incidence Study. Soc Psychiatry Psychiatr Epidemiol. 2010;45:513-21.

5. Moos RH, Cronkite RC. Symptom-based predictors of a 10-year chronic course of treated depression. J Nerv Ment Dis. 1999;187:360-8.

6. Maes M, Lambrechts J, Bosmans E, Jacobs J, Suy E, Vandervorst C, et al. Evidence for a systemic immune activation during depression: results of leukocyte enumeration by flow cytometry in conjunction with monoclonal antibody staining. Psychol Med. 1992;22:45-53.

7. Maes M, Meltzer HY, Stevens W, Calabrese J, Cosyns P. Natural killer cell activity in major depression: relation to circulating natural killer cells, cellular indices of the immune response, and depressive phenomenology. Prog Neuropsychopharmacol Biol Psychiatry. 1994;18:717-30.

8. Rothermundt $M$, Arolt V, Arolt V, Peters M, Gutbrodt H, Fenker J, et al. Inflammatory markers in major depression and melancholia. J Affect Disord. 2001;63:93-102.

9. Miller $\mathrm{AH}$, Raison $\mathrm{CL}$. The role of inflammation in depression: from evolutionary imperative to modern treatment target. Nat Rev Immunol. 2016;16:22-34.

10. Hodes GE, Pfau ML, Leboeuf M, Golden SA, Christoffel DJ, Bregman D, et al. Individual differences in the peripheral immune system promote resilience versus susceptibility to social stress. Proc Natl Acad Sci USA. 2014;111:16136-41.

11. Whitton AE, Treadway MT, Pizzagalli DA. Reward processing dysfunction in major depression, bipolar disorder and schizophrenia. Curr Opin Psychiatry. 2015;28:7-12.

12. Capuron L, Miller AH. Cytokines and psychopathology: lessons from interferonalpha. Biol Psychiatry. 2004;56:819-24.

13. Raison CL, Demetrashvili M, Capuron L, Miller AH. Neuropsychiatric adverse effects of interferon-alpha: recognition and management. CNS Drugs. 2005;19:105-23.

14. Dowlati Y, Herrmann N, Swardfager W, Liu H, Sham L, Reim EK, et al. A metaanalysis of cytokines in major depression. Biol Psychiatry. 2010;67:446-57.

15. Howren MB, Lamkin DM, Suls J. Associations of depression with C-reactive protein, IL-1, and IL-6: a meta-analysis. Psychosom Med. 2009;71:171-86.

16. Liu Y, Ho RC-M, Mak A. Interleukin (IL)-6, tumour necrosis factor alpha (TNF-a) and soluble interleukin-2 receptors (sIL-2R) are elevated in patients with major depressive disorder: a meta-analysis and meta-regression. J Affect Disord. 2012;139:230-9.

17. Slavich GM, Irwin MR. From stress to inflammation and major depressive disorder: a social signal transduction theory of depression. Psychol Bull. 2014;140:774-815.

18. Danese A, Moffitt TE, Pariante CM, Ambler A, Poulton R, Caspi A. Elevated inflammation levels in depressed adults with a history of childhood maltreatment. Arch Gen Psychiatry. 2008;65:409-15.

19. Kiecolt-Glaser JK, Gouin JP, Weng NP, Malarkey WB, Beversdorf DQ, Glaser R. Childhood adversity heightens the impact of later-life caregiving stress on telomere length and inflammation. Psychosom Med. 2011;73:16-22.

20. Miller $\mathrm{AH}$, Haroon $\mathrm{E}$, Raison $\mathrm{CL}$, Felger JC. Cytokine targets in the brain: impact on neurotransmitters and neurocircuits. Depress Anxiety. 2013;30:297-306.

21. Felger JC, Lotrich FE. Inflammatory cytokines in depression: neurobiological mechanisms and therapeutic implications. Neuroscience. 2013;246:199-229.

22. Alexander C, Rietschel ET. Bacterial lipopolysaccharides and innate immunity. J Endotoxin Res. 2001;7:167-202.

23. Lasselin J, Treadway MT, Lacourt TE, Soop A, Olsson MJ, Karshikoff B, et al. Lipopolysaccharide alters motivated behavior in a monetary reward task: a randomized trial. Neuropsychopharmacology. 2017;42:801-10.

24. Kronfol Z, Silva J Jr, Greden J, Dembinski S, Gardner R, Carroll B. Impaired lymphocyte function in depressive illness. Life Sci. 1983;33:241-7.

25. Pizzagalli DA, Jahn AL, O'Shea JP. Toward an objective characterization of an anhedonic phenotype: a signal-detection approach. Biol Psychiatry. 2005;57:319-27.

26. Pizzagalli DA, losifescu D, Hallett LA, Ratner KG, Fava M. Reduced hedonic capacity in major depressive disorder: evidence from a probabilistic reward task. J Psychiatr Res. 2008;43:76-87.

27. Knutson B, Adams CM, Fong GW, Hommer D. Anticipation of increasing monetary reward selectively recruits nucleus accumbens. J Neurosci. 2001;21:RC159.

28. Knutson B, Fong GW, Adams CM, Varner JL, Hommer D. Dissociation of reward anticipation and outcome with event-related fMRI. Neuroreport. 2001;12:3683-7.

29. Stern ER, Welsh RC, Fitzgerald KD, Gehring WJ, Lister JJ, Himle JA, et al. Hyperactive error responses and altered connectivity in ventromedial and frontoinsular cortices in obsessive-compulsive disorder. Biol Psychiatry. 2011;69:583-91.

30. First MB, Williams JBW, Karg RS, Spitzer RL. Structured Clinical Interview for DSM-5 - Research Version (SCID-5 for DSM-5, Research Version; SCID-5-RV). Arlington, VA: American Psychiatric Association; 2015.

31. Shelton RC, Falola M, Li L, Zajecka J, Fava M, Papakostas GI. The pro-inflammatory profile of depressed patients is (partly) related to obesity. J Psychiatr Res. 2015;70:91-7.

32. Khaodhiar L, Ling P-R, Blackburn GL, Bistrian BR. Serum levels of interleukin- 6 and $C$-reactive protein correlate with body mass index across the broad range of obesity. JPEN J Parenter Enter Nutr. 2004;28:410-5.

33. Malavazos AE, Corsi MM, Ermetici F, Coman C, Sardanelli F, Rossi A, et al. Proinflammatory cytokines and cardiac abnormalities in uncomplicated obesity: relationship with abdominal fat deposition. Nutr Metab Cardiovasc Dis. 2007;17:294-302.

34. Franceschi $C$, Campisi J. Chronic inflammation (inflammaging) and its potential contribution to age-associated diseases. J Gerontol A, Biol Sci Med Sci. 2014;69 (Suppl 1):S4-9. 
35. Felger JC, Haroon E, Patel TA, Goldsmith DR, Wommack EC, Woolwine BJ, et al. What does plasma CRP tell us about peripheral and central inflammation in depression? Mol Psychiatry. 2018. https://doi.org/10.1038/s41380-0180096-3.

36. Oertelt-Prigione $S$. The influence of sex and gender on the immune response. Autoimmun Rev. 2012;11:A479-85.

37. Jha MK, Minhajuddin A, Chin-Fatt C, Greer TL, Carmody TJ, Trivedi MH. Sex differences in the association of baseline c-reactive protein (CRP) and acutephase treatment outcomes in major depressive disorder: findings from the EMBARC study. J Psychiatr Res. 2019;113:165-71.

38. Snaith RP, Hamilton M, Morley S, Humayan A, Hargreaves D, Trigwell P. A scale for the assessment of hedonic tone the Snaith-Hamilton Pleasure Scale. Br J Psychiatry. 1995;167:99-103.

39. Gard DE, Kring AM, Gard MG, Horan WP, Green MF. Anhedonia in schizophrenia: distinctions between anticipatory and consummatory pleasure. Schizophr Res. 2007;93:253-60

40. Kundu $P$, Inati SJ, Evans JW, Luh W-M, Bandettini PA. Differentiating BOLD and non-BOLD signals in $\mathrm{fMRI}$ time series using multi-echo EPI. Neuroimage. 2012;60:1759-70.

41. Kundu $P$, Brenowitz ND, Voon V, Worbe $Y$, Vértes $P E$, Inati SJ, et al. Integrated strategy for improving functional connectivity mapping using multiecho fMRI. Proc Natl Acad Sci USA. 2013:110:16187-92.

42. Kundu P, Voon V, Balchandani P, Lombardo MV, Poser BA, Bandettini PA. Multiecho fMRI: A review of applications in $\mathrm{fMRI}$ denoising and analysis of BOLD signals. Neuroimage. 2017;154:59-80.

43. Sundkvist A, Myte R, Bodén S, Enroth S, Gyllensten U, Harlid S, et al. Targeted plasma proteomics identifies a novel, robust association between cornulin and Swedish moist snuff. Sci Rep. 2018;8:2320.

44. van Buuren S, Groothuis-Oudshoorn K. mice: Multivariate imputation by chained equations in R. J Stat Softw. 2011;45. https://doi.org/10.18637/jss.v045.i03.

45. Heitjan DF, Little JA. Multiple imputation for the fatal accident reporting system. J R Stat Soc Ser C, Appl Stat. 1991.

46. Schenker N, Taylor JMG. Partially parametric techniques for multiple imputation. Comput Stat Data Anal. 1996;22:425-46.

47. Marshall A, Altman DG, Holder RL. Comparison of imputation methods for handling missing covariate data when fitting a Cox proportional hazards model: a resampling study. BMC Med Res Methodol. 2010;10:112.

48. Atlases - FslWiki. 2019. https://fsl.fmrib.ox.ac.uk/fsl/fslwiki/Atlases.

49. Cox RW, Chen G, Glen DR, Reynolds RC, Taylor PA. fMRI clustering and falsepositive rates. Proc Natl Acad Sci USA. 2017;114:E3370-1.

50. Moses-Kolko EL, Fraser D, Wisner KL, James JA, Saul AT, Fiez JA, et al. Rapid habituation of ventral striatal response to reward receipt in postpartum depression. Biol Psychiatry. 2011;70:395-9.

51. Vogelzangs $\mathrm{N}$, de Jonge $\mathrm{P}, \mathrm{Smit} \mathrm{JH}$, Bahn S, Penninx BW. Cytokine production capacity in depression and anxiety. Transl Psychiatry. 2016;6:e825.

52. Freed RD, Mehra LM, Laor D, Patel M, Alonso CM, Kim-Schulze $S$, et al. Anhedonia as a clinical correlate of inflammation in adolescents across psychiatric conditions. World J Biol Psychiatry. 2018;20:1-11.

53. Phillips ML, Swartz HA. A critical appraisal of neuroimaging studies of bipolar disorder: toward a new conceptualization of underlying neural circuitry and a road map for future research. Am J Psychiatry. 2014;171:829-43.

54. Zhang H-X, Xu YQ, Li YY, Lu MF, Shi SX, Ji JL, et al. Difference in proinflammatory cytokines produced by monocytes between patients with major depressive disorder and healthy controls. J Affect Disord. 2018;234:305-10.

55. Köhler CA, Freitas TH, Maes M, de Andrade NQ, Liu CS, Fernandes BS, et al. Peripheral cytokine and chemokine alterations in depression: a meta-analysis of 82 studies. Acta Psychiatr Scand. 2017;135:373-87.

56. Goldsmith DR, Rapaport MH, Miller BJ. A meta-analysis of blood cytokine network alterations in psychiatric patients: comparisons between schizophrenia, bipolar disorder and depression. Mol Psychiatry. 2016;21:1696-709.

57. Chamberlain SR, Cavanagh J, de Boer P, Mondelli V, Jones D, Drevets WC, et al. Treatment-resistant depression and peripheral C-reactive protein. $\mathrm{Br} J$ Psychiatry. 2019;214:11-9.

58. Syed SA, Beurel E, Loewenstein DA, Lowell JA, Craighead WE, Dunlop BW, et al. Defective inflammatory pathways in never-treated depressed patients are associated with poor treatment response. Neuron. 2018;99:914-24. e3.

59. Cathomas F, Klaus F, Guetter K, Chung HK, Raja Beharelle A, Spiller TR, et al. Increased random exploration in schizophrenia is associated with inflammation. NPJ Schizophr. 2021;7:6

60. Eyre HA, Air T, Pradhan A, Johnston J, Lavretsky H, Stuart MJ, et al. A metaanalysis of chemokines in major depression. Prog Neuropsychopharmacol Biol Psychiatry. 2016;68:1-8

61. Moylan S, Maes M, Wray NR, Berk M. The neuroprogressive nature of major depressive disorder: pathways to disease evolution and resistance, and therapeutic implications. Mol Psychiatry. 2013;18:595-606.
62. Stuart MJ, Singhal G, Baune BT. Systematic review of the neurobiological relevance of chemokines to psychiatric disorders. Front Cell Neurosci. 2015;9:357.

63. Weiss JM, Downie SA, Lyman WD, Berman JW. Astrocyte-derived monocytechemoattractant protein-1 directs the transmigration of leukocytes across a model of the human blood-brain barrier. J Immunol. 1998;161:6896-903.

64. Suarez EC, Lewis JG, Krishnan RR, Young KH. Enhanced expression of cytokines and chemokines by blood monocytes to in vitro lipopolysaccharide stimulation are associated with hostility and severity of depressive symptoms in healthy women. Psychoneuroendocrinology. 2004;29:1119-28.

65. Felger JC, Li Z, Haroon E, Woolwine BJ, Jung MY, Hu X, et al. Inflammation is associated with decreased functional connectivity within corticostriatal reward circuitry in depression. Mol Psychiatry. 2016;21:1358-65.

66. Harrison NA, Brydon L, Walker C, Gray MA, Steptoe A, Critchley HD. Inflammation causes mood changes through alterations in subgenual cingulate activity and mesolimbic connectivity. Biol Psychiatry. 2009;66:407-14.

67. Eisenberger NI, Berkman ET, Inagaki TK, Rameson LT, Mashal NM, Irwin MR. Inflammation-induced anhedonia: endotoxin reduces ventral striatum responses to reward. Biol Psychiatry. 2010;68:748-54.

68. Capuron L, Pagnoni G, Drake DF, Woolwine BJ, Spivey JR, Crowe RJ, et al. Dopaminergic mechanisms of reduced basal ganglia responses to hedonic reward during interferon alfa administration. Arch Gen Psychiatry. 2012;69:1044-53.

69. Wise RA. Brain reward circuitry: insights from unsensed incentives. Neuron 2002;36:229-40.

70. Pratt DN, Barch DM, Carter CS, Gold JM, Ragland JD, Silverstein SM, et al. Reliability and replicability of implicit and explicit reinforcement learning paradigms in people with psychotic disorders. Schizophr Bull. 2020. https://doi.org/10.1093/ schbul/sbaa165.

71. Boyle CC, Kuhlman KR, Dooley LN, Haydon MD, Robles TF, Ang YS, et al Inflammation and dimensions of reward processing following exposure to the influenza vaccine. Psychoneuroendocrinology. 2019;102:16-23.

\section{ACKNOWLEDGEMENTS}

Research reported in this publication was supported by the National Institute of Mental Health of the National Institutes of Health under Award Number R21MH109771 to JWM. DAP was partially supported by NIMH grant R37 MH068376. AEW received support from the National Health and Medical Research Council, Grant No. APP1110773. The content is solely the responsibility of the authors and does not necessarily represent the official views of the National Institutes of Health. Additional funding for this study was provided by the Ehrenkranz Laboratory for Human Resilience, a component of the Depression and Anxiety Center for Discovery and Treatment at the Icahn School of Medicine at Mount Sinai and by the Gottesman Foundation.

\section{AUTHOR CONTRIBUTIONS}

JWM, SR, ERS, and SC conceived the experiment; SC, AC, MP, HX, and KAC collected the data; SC, LSM, NFF, KSS, FC, MKP, AEW, and DAP analyzed the data; SC, LSM, FC, and JWM wrote the manuscript.

\section{COMPETING INTERESTS}

In the past 5 years, Dr. Murrough has provided consultation services and/or served on advisory boards for Allergan, Boehreinger Ingelheim, Clexio Biosciences, Fortress Biotech, FSV7, Global Medical Education (GME), Otsuka, and Sage Therapeutics. Dr. Murrough is named on a patent pending for neuropeptide $Y$ as a treatment for mood and anxiety disorders and on a patent pending for the use of ezogabine and other KCNQ channel openers to treat depression and related conditions. The Icahn School of Medicine (employer of Dr. Murrough) is named on a patent and has entered into a licensing agreement and will receive payments related to the use of ketamine or esketamine for the treatment of depression. The Icahn School of Medicine is also named on a patent related to the use of ketamine for the treatment of PTSD. Dr. Murrough is not named on these patents and will not receive any payments. Dr. Collins is a paid independent rater for Medavante-Prophase. Dr. Costi has provided consultation services for Guidepoint. Over the past 3 years, Dr. Pizzagalli has received consulting fees from Akili Interactive Labs, BlackThorn Therapeutics, Boehreinger Ingelheim, Compass Pathway, Posit Science, Otsuka Pharmaceuticals, and Takeda Pharmaceuticals; one honorarium from Alkermes; stock options from BlackThorn Therapeutics; and funding from NIMH, Brain and Behavior Research Foundation, the Dana Foundation, and Millennium Pharmaceuticals. Dr. Pizzagalli has a financial interest in BlackThorn Therapeutics, which has licensed the copyright to the Probabilistic Reward Task through Harvard University. Dr. Pizzagalli's interests were reviewed and are managed by McLean Hospital and Partners HealthCare in 


\section{8}

accordance with their conflict of interest policies. All the other authors report no financial relationships with commercial interests.

\section{ADDITIONAL INFORMATION}

Supplementary information The online version contains supplementary material available at https://doi.org/10.1038/s41398-021-01668-1.

Correspondence and requests for materials should be addressed to James W. Murrough.

Reprints and permission information is available at http://www.nature.com/ reprints

Publisher's note Springer Nature remains neutral with regard to jurisdictional claims in published maps and institutional affiliations. (i) Open Access This article is licensed under a Creative Commons Attribution 4.0 International License, which permits use, sharing, adaptation, distribution and reproduction in any medium or format, as long as you give appropriate credit to the original author(s) and the source, provide a link to the Creative Commons license, and indicate if changes were made. The images or other third party material in this article are included in the article's Creative Commons license, unless indicated otherwise in a credit line to the material. If material is not included in the article's Creative Commons license and your intended use is not permitted by statutory regulation or exceeds the permitted use, you will need to obtain permission directly from the copyright holder. To view a copy of this license, visit http://creativecommons. org/licenses/by/4.0/.

This is a U.S. government work and not under copyright protection in the U.S.; foreign copyright protection may apply 2021 